\title{
Nanopore Formation and Phosphatidylserine Externalization in a Phospholipid Bilayer at High Transmembrane Potential
}

\author{
P. Thomas Vernier, Matthew J. Ziegler, Yinghua Sun, Wenji V. Chang, Martin A. Gundersen, and D. \\ Peter Tieleman \\ Departments of Electrical Engineering and Chemical Engineering and Materials Science, Viterbi School of \\ Engineering (VSE), University of Southern California (USC), Los Angeles CA, 90089-0271 and Department of \\ Biological Sciences, University of Calgary, Calgary, Alberta, Canada T2N 1N4
}

\section{Supporting Information}

\section{Methods}

Cell lines and culture conditions. Human RPMI 8226 multiple myeloma cells (ATCC CCL-155) were grown in RPMI 1640 medium (Irvine Scientific, Irvine, CA) containing 10\% heat-inactivated fetal bovine serum (FBS; Gibco, Carlsbad, CA), $2 \mathrm{mM}$ L-glutamine (Gibco, Carlsbad, CA), 50 units $/ \mathrm{mL}$ penicillin (Gibco, Carlsbad, CA), and $50 \mu \mathrm{g} / \mathrm{mL}$ streptomycin (Gibco, Carlsbad, CA) at $37 \mathrm{C}$ in a humidified, $5 \%$ carbon dioxide atmosphere.

Pulse generators and pulse exposures. For microscopic observations, cells were placed in a rectangular channel $100 \mu \mathrm{m}$ wide, $30 \mu \mathrm{m}$ deep, and $12 \mathrm{~mm}$ long, with gold-plated electrode walls, microfabricated with photolithographic methods on a glass microscope slide. A USC-designed and fabricated NanoPulser with fast recovery diode switching was mounted on the microscope stage for delivery of $4 \mathrm{~ns}$ pulses directly to the microchamber electrodes in ambient atmosphere at room temperature ${ }^{1}$.

Fluorescence microscopy. Observations of live cells during pulse exposures were made with a Zeiss Axiovert 200 epifluorescence microscope. For visualization of PS translocation with FM1-43 (Molecular Probes, Eugene OR; $\lambda_{\text {ex }}=480$ $\mathrm{nm}, \lambda_{\mathrm{em}}=535 \mathrm{~nm}$ ), cells were washed twice, resuspended in growth medium containing $20 \mu \mathrm{M}$ FM1-43, incubated for 20 minutes at $37{ }^{\circ} \mathrm{C}$, and observed without additional washing. Real-time binding of annexin $\mathrm{V}$ was observed using reagents from an apoptosis detection kit from Oncogene Research Products (San Diego). Cells were suspended in RPMI 1640 medium containing $2.5 \mathrm{mM} \mathrm{CaCl}_{2}$ and incubated before pulse exposure with media binding reagent and annexin V-FITC for $15 \mathrm{~min}$ in the dark according to the manufacturer's instructions, except that in order to increase the sensitivity of the fluorescence microscopic imaging the annexin $\mathrm{V}$ concentration was 16 times the value recommended for flow cytometric analysis. Images were captured and analyzed with a LaVision Imager QE camera and software (LaVision, Goettingen, Germany).

Molecular dynamics simulations. The bilayer test system consists of 128 lipids, 2873 Simple Point Charge water molecules $^{2}$, and 4 sodium ions. One of the two leaflets of the lipid bilayer includes 4 dioleoylphosphatidylserine (DOPS) molecules and 60 dioleoylphosphatidylcholine molecules (DOPC). The other bilayer leaflet contains 64 DOPC molecules. $\mathrm{CH}_{2}$ and $\mathrm{CH}_{3}$ groups are treated as a united atom. Test systems were energy minimized using a steepest descent algorithm and then equilibrated for $25 \mathrm{~ns}$. Production simulations with applied electric fields were run for times from 1 to 25 ns with a time step of 2 fs using periodic boundary conditions. Bond lengths were constrained using LINCS ${ }^{3}$ for lipids and SETTLE $^{4}$ for water. Electrostatic interactions were calculated with a Particle Mesh Ewald algorithm ${ }^{5}$ using fast Fourier transforms with a cutoff radius of $1 \mathrm{~nm}$ for coulombic and Lennard-Jones interactions. Solvent, DOPC, DOPS, and ions were coupled separately to temperature baths of $300 \mathrm{~K}$ with a coupling constant of $0.1 \mathrm{ps}^{6}$. Pressure was maintained at $1 \mathrm{bar}$ also using a Berendsen algorithm with a relaxation time of $1 \mathrm{ps}$. The system size was allowed to fluctuate anisotropically with a compressibility of $4.5 \times 10^{-5} / \mathrm{bar}$ in the $\mathrm{z}$ direction and 0 in the $\mathrm{x}$ and $\mathrm{y}$ directions to maintain the bilayer shape throughout the simulation. Systems were subjected to an external electric field from $0.2 \mathrm{~V} / \mathrm{nm}$ and $0.8 \mathrm{~V} / \mathrm{nm}$. The phospholipid parameters were derived from the OPLS-united atom based parameters of Berger et al. ${ }^{7}$ with additional parameters for the PS headgroup from Mukhopadhyay et al. ${ }^{8}$ and newly derived charges [ $\mathrm{Zu}$ et al., in preparation]. The behavior of DOPC and POPS has been well-tested, and minor differences in the PS head group for this study are not important. All simulations were run using GROMACS version 3.2.1 $1^{9,10}$ on the University of Southern California High Performance Computing and Communications Linux cluster (http://www.usc.edu/hpcc/). Molecular graphics images were generated with $\mathrm{VMD}^{11}$. 


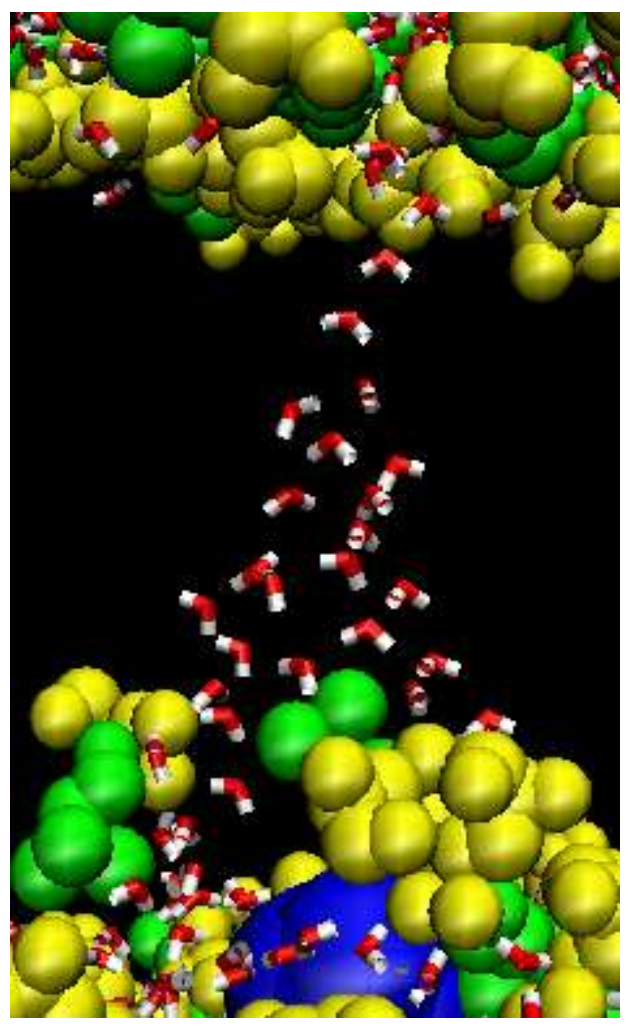

Figure S1. Water dipoles aligned in the low dielectric permittivity of the membrane interior during the formation of a hydrophilic pore. This is an enlarged view of the center of Fig. $2 \mathrm{~b}$ (simulation time $5.5 \mathrm{~ns}$ ). The field direction is from top to bottom, with the positive electrode at the top.

\section{References}

1. Kuthi, A.; Gabrielsson, P.; Behrend, M.; Vernier, P. T.; Gundersen, M., Nanosecond pulse generator using fast recovery diodes for cell electromanipulation. IEEE Trans. on Plasma Sci. 2005, 33, (4), 1192-1197.

2. Berendsen, H. J. C.; Postma, J. P. M.; van Gunsteren, W. F.; Hermans, J., Interaction models for water in relation to protein hydration. In Intermolecular Forces, ed. Pullman, B., Reidel, Dordrecht, Netherlands, 1981, pp 331-342.

3. Hess, B.; Bekker, H.; Berendsen, H. J. C.; Fraaije, J. G. E. M., LINCS: A linear constraint solver for molecular simulations. Journal of Computational Chemistry 1997, 18, (12), 1463-1472.

4. Miyamoto, S.; Kollman, P. A., Settle - an analytical version of the shake and rattle algorithm for rigid water models. Journal of Computational Chemistry 1992, 13, (8), 952-962.

5. Essmann, U.; Perera, L.; Berkowitz, M. L.; Darden, T.; Lee, H.; Pedersen, L. G., A smooth particle mesh Ewald method. Journal of Chemical Physics 1995, 103, (19), 8577-8593.

6. Berendsen, H. J. C.; Postma, J. P. M.; van Gunsteren, W. F.; Dinola, A.; Haak, J. R., Molecular dynamics with coupling to an external bath. Journal of Chemical Physics 1984, 81, (8), 3684-3690.

7. Berger, O.; Edholm, O.; Jahnig, F., Molecular dynamics simulations of a fluid bilayer of dipalmitoylphosphatidylcholine at full hydration, constant pressure, and constant temperature. Biophys $J$ 1997, 72, (5), 2002-13.

8. Mukhopadhyay, P.; Monticelli, L.; Tieleman, D. P., Molecular dynamics simulation of a palmitoyl-oleoyl phosphatidylserine bilayer with $\mathrm{Na}+$ counterions and $\mathrm{NaCl}$. Biophys $J$ 2004, 86, (3), 1601-9.

9. Berendsen, H. J. C.; van der Spoel, D.; van Drunen, R., Gromacs - a message-passing parallel molecular-dynamics implementation. Computer Physics Communications 1995, 91, (1-3), 43-56.

10. Lindahl, E.; Hess, B.; van der Spoel, D., GROMACS 3.0: a package for molecular simulation and trajectory analysis. Journal of Molecular Modeling 2001, 7, (8), 306-317.

11. Humphrey, W.; Dalke, A.; Schulten, K., VMD: visual molecular dynamics. J Mol Graph 1996, 14, (1), 33-8, 27-8. 